\title{
Molecular theory of proper ferroelectricity in bent-core liquid crystals
}

\author{
M.A. Osipov ${ }^{1}$ and G. Pająk ${ }^{2, a}$ \\ 1 Department of Mathematics and Statistics, University of Strathclyde, Livingstone Tower, Richmond Street, \\ Glasgow, Scotland, UK \\ 2 Faculty of Physics, Mathematics and Computer Science, Tadeusz Kościuszko Cracow University of Technology, \\ Podchorążych 1, Kraków, Poland
}

Received 2 May 2014 and Received in final form 4 August 2014

Published online: 12 September 2014

(C) The Author(s) 2014. This article is published with open access at Springerlink.com

\begin{abstract}
Antiferro- and ferro-electric ordering has been discovered in orthogonal smectic phases composed of nonchiral bent-core molecules. These systems are the only proper fluid ferroelectrics confirmed experimentally so far. We consider a molecular theory of proper ferroelectric ordering in isotropic, nematic and smectic A phases and conclude that the delicate balance between the tendencies for local parallel and antiparallel ordering of molecular electric and steric dipoles is strongly shifted in restricted geometries. This is a reason why dipolar ordering is more likely to occur within a smectic layer. We derive model interaction potentials for polar bent-core molecules and present the results of the mean-field theory of ferroelectric ordering in the orthogonal smectic phase taking into account also the molecular biaxiality. Order parameter profiles have been calculated numerically and phase diagrams are presented which enable one to analyze the relative importance of dipole-dipole interaction and intermolecular attraction modulated by polar bent-core molecular shape.
\end{abstract}

\section{Introduction}

Ferroelectric ordering in chiral tilted smectic Liquid Crystals (LCs) has been studied for decades. One notes, however, that these systems are characterized by the so-called improper ferroelectricity which means that the spontaneous polarization is not a primary order parameter (that is it does not appear self-consistently), but is induced by the tilt in the chiral medium [1]. From the microscopic point of view the ferroelectric ordering in chiral smectics $\mathrm{C}^{*}$ is not determined by electrostatic dipole-dipole interactions but has a more complicated origin because the ordering of molecular dipoles is induced by a nonpolar order of chiral molecules. The corresponding intermolecular interactions are discussed in detail in [2].

In contrast, in proper ferroelectric materials polarization is the primary order parameter and the transition into the polar phase is determined by polar intermolecular interactions including, in particular, the dipole-dipole ones. In principle, ferroelectric ordering may occur spontaneously also in isotropic and nematic phases composed of strongly polar molecules. Such a transition into the ferroelectric isotropic and/or nematic phase is indeed predicted by simple mean-field theories $[3-6]$. On the other

\footnotetext{
${ }^{a}$ e-mail: grzegorz@th.if.uj.edu.pl
}

hand, polar phases of this kind have never been observed experimentally (at least the spontaneous polarization has not been measured directly) except for more complicated columnar systems. One notes, however, that strong experimental evidence has recently been presented in favor of proper ferroelectric ordering in some polymers nematic LCs [7]. From the theoretical point of view the dipoledipole interaction must be very strong in order to induce ferroelectric ordering in a fluid [4]. On the other hand, molecules with very large permanent dipoles have a strong tendency to form polar chains (see ref. [4] and references therein) which is generally a more efficient way to reduce the total free energy then the transition into the homogeneous ferroelectric phase in the bulk [4]. As discussed in more detail in sect. 2 , this is one of the reasons why proper ferroelectricity is not widely observed in strongly polar fluids.

Recently proper antiferro- [8-12] and ferro-electric [13] ordering has finally been discovered in orthogonal smectic phases composed of nonchiral bent-core molecules. Orthogonal bent-core smectics are the only proper fluid ferroelectrics completely confirmed experimentally so far. As observed in ref. [13], the spontaneous polarization in the ferroelectric bent-core smectic phase appears to be very large which is consistent with large values of the orientational order parameter reported very recently in [14]. 
One notes that bent-core LCs exhibit a number of smectic phases with periodicity of more then one layer including the antiferroelectric orthogonal phase, random, and possibly, the three-layer phase [15]. A phenomenological theory of bent-core smectic phases with such periodicity has recently been developed [16,17], but at present there is no consistent molecular theory of ferroelectric ordering in orthogonal bent-core smectics and even the microscopic mechanism of such an ordering in not understood in detail.

In this paper we first discuss in sect. 2 the general results of the molecular theory of proper ferroelectric ordering in isotropic, nematic and smectic A phases and conclude that the delicate balance between the tendencies for local parallel and antiparallel ordering of molecular electric and steric dipoles is strongly shifted in restricted geometries. This is a reason why dipolar ordering is more likely to occur within a smectic layer then in the homogeneous nematic phase composed of the same molecules. In sect. 3 we derive model interaction potentials for polar bent-core molecules, and in sect. 4 we present the results of the mean-field theory of ferroelectric ordering in the orthogonal smectic phase taking into account also the molecular biaxiality. Finally we conclude the paper by emphasizing the most important outcomes and outline possible future issues.

\section{Dipole-dipole interaction and polar ordering in complex fluids}

It is often assumed that the ferroelectric ordering in smectic phases composed of bent-core molecules is primarily determined by the close packing of bent cores. One notes, however, that packing entropy generally does not play a leading role in thermotropic liquid crystal [18] because the corresponding contribution to the total free energy is relatively small. Indeed, in the mean-field approximation the packing entropy of the nematic phase can be expressed as

$$
\begin{aligned}
S_{p}= & \frac{1}{2} \rho^{2} k_{B} T \int\left[\theta\left(r_{12}-\xi_{12}\right)\right. \\
& -1] f\left(\omega_{1}\right) f\left(\omega_{2}\right) \mathrm{d}^{3} \mathbf{r}_{12} \mathrm{~d} \omega_{1} \mathrm{~d} \omega_{2},
\end{aligned}
$$

where $\rho$ is the number density, $f(\omega)$ is the orientational distribution function which depends on molecular orientational coordinates $\omega$ of one particle, and $\theta\left(r_{12}-\xi_{12}\right)$ is the step-function which vanishes if the molecules " 1 " and " 2 " penetrate each other and which is equal to 1 otherwise. Here $\xi_{12}$ is the closest distance of approach for the two molecules at fixed relative orientation. One can readily see from eq. (1) that the packing entropy is of the order $\rho^{2} k_{B} T$. At the same time the mean-field contribution from the intermolecular attraction is of the order of $\rho^{2} V_{0}$, where $V_{0}$ is the value of the total interaction potential at average intermolecular separation. The energy $V_{0}$, however, is typically $5-10$ times larger than $k_{B} T_{N I}$ [18], where $T_{N I}$ is the Isotropic-Nematic transition temperature, and thus the predominant contribution to the free energy is determined by the intermolecular attraction modulated by anisotropic molecular shape [19].
Bent-core molecules which exhibit the ferroelectric smectic phase possess significant transverse dipoles, and thus a contribution from the dipole-dipole interaction to the stability of the polar phase cannot be neglected. Indeed, it has been shown by atomistic simulations that polar clusters in the bent-core nematic phase disappear when electrostatic interaction is "switched off" [20]. One may conclude that in real ferroelectric bent-core smectics both dipole-dipole interactions and the effects of polar shape should be taken into consideration. Recently steric polar interactions of $\mathrm{V}$-shaped molecules have been considered in detail by Bisi et al. [21,22]. At the same time, in the existing molecular-statistical theory [3-6] the dipole-dipole interaction is considered to be responsible for ferroelectric ordering in isotropic fluids composed of strongly polar but weakly anisometric molecules. However, as discussed in the Introduction, there is no direct experimental evidence if favor of ferroelectricity in isotropic fluids. There are several reasons for that which are discussed in [4]. Firstly, the fluid ferroelectric phase is stable only if the molecular dipole is sufficiently large. On the other hand, in such systems there is a strong tendency to form polar chains which appears to be a more effective way to reduce the total free energy (see, for example, [23]). The polar interaction between such chains, however, appears to be relatively weak and they do not order ferroelectrically.

Secondly, the contribution of the long-range dipoledipole interaction to the free energy of a sample generally depends on the boundary conditions and therefore the conditions, which determine the transition temperature into the ferroelectric phase, are not universal [4]. They may depend, for example, on the sample shape and on the dielectric susceptibility of the surrounding medium. In this section we show that this problem does not exist in quasi-two-dimensional systems, i.e. in the $2 \mathrm{D}$ confined geometry. Thus ferroelectricity is more likely to be found in layered systems like smectic liquid crystals.

In the molecular-field approximation the average dipole-dipole interaction energy in the polar fluid composed of spherical molecules with permanent dipoles can be expressed as

$$
\begin{aligned}
\left\langle U_{d d}\right\rangle= & \frac{1}{2} \rho^{2} \int \theta\left(r_{i j}-D\right) \phi_{d d}\left(\mathbf{a}_{i}, \mathbf{a}_{j}\right) f\left(\mathbf{a}_{i}\right) f\left(\mathbf{a}_{j}\right) \\
& \times r_{i j}^{2} \mathrm{~d} r_{i j} \mathrm{~d}^{2} \mathbf{u}_{i j} \mathrm{~d} \mathbf{a}_{i} \mathrm{~d} \mathbf{a}_{j}
\end{aligned}
$$

where $\phi_{d d}\left(\mathbf{a}_{i}, \mathbf{a}_{j}\right)$ is the electrostatic dipole-dipole interaction potential:

$$
\phi_{d d}\left(\mathbf{a}_{i}, \mathbf{a}_{j}\right)=\frac{1}{r_{i j}^{3}}\left(\vec{\mu}_{i} \cdot \vec{\mu}_{j}-3\left(\vec{\mu}_{i} \cdot \vec{u}_{i j}\right)\left(\vec{\mu}_{j} \cdot \vec{u}_{i j}\right)\right)
$$

and where $\vec{\mu}_{i}$ is the permanent dipole of the molecule $i$, $\mathbf{a}_{i}$ is the unit vector along the molecular dipole, $D$ is the molecular diameter, $\mathbf{u}_{i j}=\mathbf{r}_{i j} / r_{i j}$ is the unit intermolecular vector and $f\left(\mathbf{a}_{i}\right)$ is one-particle orientational distribution function. One can readily see that the integral over $\mathbf{u}_{i j}$ in eq. (2) vanishes because $\int u_{\alpha} u_{\beta} \mathrm{d}^{2} \mathbf{u}=4 \pi \delta_{\alpha \beta} / 3$. At the same time the integral over $r_{i j}$ diverges as $\ln L$, where 
$L$ is the system size. Thus the actual value of the dipoledipole contribution to the total free energy depends on the shape of the sample and on boundary conditions [4].

The explicit dependence of the integral on the electrostatic boundary conditions can be seen in the following way. Let us express the integral in eq. (2) as a sum of the following two parts:

$$
\begin{aligned}
\left\langle U_{d d}\right\rangle= & \frac{1}{2} \rho^{2} \int_{V_{0}}\left[\theta\left(r_{i j}-D\right)\right. \\
& -1] \phi_{d d}\left(\mathbf{a}_{i}, \mathbf{a}_{j}\right) f\left(\mathbf{a}_{i}\right) f\left(\mathbf{a}_{j}\right) r_{i j}^{2} \mathrm{~d} r_{i j} \mathrm{~d}^{2} \mathbf{u}_{i j} \mathrm{~d} \mathbf{a}_{i} \mathrm{~d} \mathbf{a}_{j} \\
& +\frac{1}{2} \rho^{2} \int \phi_{d d}\left(\mathbf{a}_{i}, \mathbf{a}_{j}\right) f\left(\mathbf{a}_{i}\right) f\left(\mathbf{a}_{j}\right) r_{i j}^{2} \mathrm{~d} r_{i j} \mathrm{~d}^{2} \mathbf{u}_{i j} \mathrm{~d} \mathbf{a}_{i} \mathrm{~d} \mathbf{a}_{j},
\end{aligned}
$$

where the first integral in taken over the spherical volume $V_{0}$ of the radius $D$ with the centre at the origin and the second integral is taken over the whole sample. The advantage of this representation is related to the fact that the first integral can be taken analytically despite a singularity at the origin. Indeed, taking into account that the dipole-dipole interaction potential can be expressed as a full gradient

$$
\frac{1}{r_{i j}^{3}}\left(\vec{\mu}_{i} \cdot \vec{\mu}_{j}-3\left(\vec{\mu}_{i} \cdot \vec{u}_{i j}\right)\left(\vec{\mu}_{j} \cdot \vec{u}_{i j}\right)\right)=\mu_{i, \alpha} \mu_{j, \beta} \nabla_{\alpha} \nabla_{\beta} \frac{1}{r_{i j}},
$$

the first integral in eq. (4) can be transformed into the surface integral (employing the Gauss theorem) which does not contain any singularity and which can be evaluated analytically [24]:

$$
\frac{1}{2} \rho^{2} \int_{V_{0}}\left[\theta\left(r_{i j}-D\right)-1\right] \phi_{d d}\left(\mathbf{a}_{i}, \mathbf{a}_{j}\right) \mathrm{d} \mathbf{a}_{i} \mathrm{~d} \mathbf{a}_{j} \mathrm{~d} V=\frac{4 \pi}{3}\langle\vec{\mu}\rangle^{2},
$$

where $\langle\vec{\mu}\rangle=\int \vec{\mu} f(\mathbf{a})$ da is the average molecular dipole in the polar phase.

It can readily be shown now that the second integral in eq. (4) is the total electrostatic energy of the whole sample [4]:

$$
\frac{1}{2} \rho^{2} \int \phi_{d d}\left(\mathbf{a}_{i}, \mathbf{a}_{j}\right) \mathrm{d} \mathbf{a}_{i} \mathrm{~d} \mathbf{a}_{j} \mathrm{~d} V=-\frac{1}{2} \int \mathbf{P}(\mathbf{r}) \cdot \mathbf{E}(\mathbf{r}) \mathrm{d}^{3} \mathbf{r},
$$

where $\mathbf{P}=\rho\langle\vec{\mu}\rangle$ is the macroscopic polarization, and where we have used the well-known relationship between the electric field $\mathbf{E}$ and the polarization $\mathbf{P}$ which follows from Maxwell equations:

$$
E_{\alpha}(\mathbf{r})=-\int T_{\alpha \beta}\left(\mathbf{r}-\mathbf{r}^{\prime}\right) P_{\beta}\left(\mathbf{r}^{\prime}\right) \mathrm{d}^{3} \mathbf{r}^{\prime}
$$

where $T_{\alpha \beta}(\mathbf{r})=r^{-3}\left(\delta_{\alpha, \beta}-3 u_{\alpha} u_{\beta}\right)$, and where $\mathbf{u}=\mathbf{r} / r$.

For an ellipsoidal sample $E=-4 \pi D(k, \epsilon) P$, where $k$ is the parameter of the ellipsoid ( $k=1 / 3$ for a sphere) and $\epsilon$ is the dielectric constant of the surrounding medium. As a result the contribution of the dipole-dipole interaction to the total free energy of the ellipsoidal sample is expressed as $[4]$

$$
\Delta F=\left\langle U_{d d}\right\rangle=2 \pi\left[2 D(k, \epsilon)-\frac{2}{3}\right] P^{2}
$$

Now there are two important cases. For a spherical sample in a conducting medium $D(1 / 3, \infty)=0$ and $\mathbf{E}=0$. Thus the dipole-dipole contribution reads

$$
\Delta F=\left\langle U_{d d}\right\rangle=-\frac{4 \pi}{3} P^{2} .
$$

One concludes that in this case the dipole-dipole interaction promotes the ferroelectric ordering. In contrast, for a spherical sample in vacuum $D(1 / 3,1)=1 / 3$ and therefore the dipole-dipole contribution vanishes because the two terms cancel each other. Thus in this case the dipoledipole interaction does not contribute to the total free energy in the first approximation.

In real polar fluids a contribution of the dipole-dipole interaction to the total free energy may vary significantly, as it might depend on the sample shape, on boundary conditions and on the concentration of ions. These dependencies are related to the long-range nature of the dipoledipole interaction in three-dimensional systems, and this is one of the main reasons why proper ferroelectricity can hardly be observed in isotropic fluids or in nematic liquid crystals [4]. On the other hand, in the restricted 2D geometry the integral of the dipole-dipole interaction does not diverge at infinity, and the corresponding contribution to the free energy is given by a regular expression which is much more robust. Indeed, assuming that the molecular centres are restricted to a plane in $3 \mathrm{D}$ (i.e. the molecules belong to a thin layer), the average dipole-dipole interaction potential in a system of spherical molecules with homogeneous polarization can be expressed as:

$$
\begin{aligned}
\left\langle U_{d d}^{2 D}\right\rangle= & \frac{1}{2} s \rho_{2 D}^{2} \int \theta\left(r_{i j}-D\right) \phi_{d d}\left(\mathbf{a}_{i}, \mathbf{a}_{j}\right) f\left(\mathbf{a}_{i}\right) \\
& \times f\left(\mathbf{a}_{j}\right) r_{i j} \mathrm{~d} r_{i j} \mathrm{~d} \phi_{i j} \mathrm{~d} \mathbf{a}_{i} \mathrm{~d} \mathbf{a}_{j} \\
= & -\frac{1}{2} \frac{s \rho_{2 D}^{2}}{D}\langle\vec{\mu}\rangle^{2},
\end{aligned}
$$

where $s$ is the total surface area of the layer and $\rho_{2 D}$ is the surface number density.

Thus the dipole-dipole contribution to the free energy of the 2D layer promotes ferroelectricity and does not depend on the sample shape or on boundary conditions. This explains qualitatively why proper ferroelectricity has been observed in the smectic A phase composed of strongly polar bent-core molecules. The corresponding expression will be used in the following section.

\section{Molecular model of bent-core molecules}

In this paper we use the effective interaction potential for the model bent-core particles which is based on the interactions between three polarizability centers and the transverse electric dipole located at the apex of molecule, fig. 1. The polarizability centers are assumed to be isotropic for simplicity, and we assume that the interaction between centers is described by the Lennard-Jones potential, which includes long-range attraction and short-range repulsion. This kind of soft interaction provides the natural distance, 


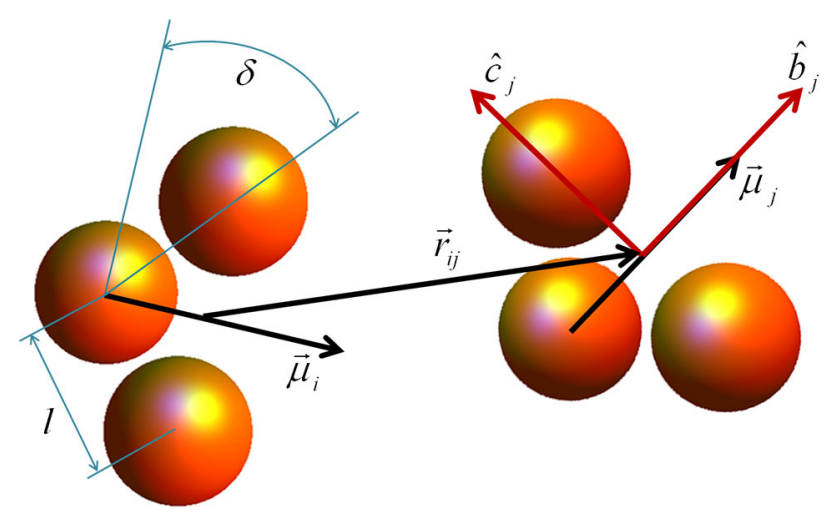

Fig. 1. (Color online) Model bent-core molecules, where the angle $\delta$ is the deviation from the uniaxial order of LennardJones centers, and two neighboring centers are located at the distance $l$. Furthermore each molecule possesses the electric dipole placed at its apex along the $C_{2 v}$ symmetry axis, which coincides with the short molecular axis $\hat{b}$. The long principal axis, $\hat{c}$, is shown for molecule $j$, and the third principal axis is determined by $\hat{a}=\hat{b} \times \hat{c}$.

i.e. $\sigma$, for the cutoff, and this is the reason why we have used them instead of the previously investigated dispersion interactions between arms [25]. Now the intermolecular interaction potential for bent-core molecules with the opening angle $\pi-2 \delta$ is expressed as a sum of LennardJones contributions and the electrostatic dipole-dipole potential:

$$
U(i, j)=\sum_{\alpha, \beta=-1,0,1} V_{L J}\left(r_{i_{\alpha}, j_{\beta}}\right)+\phi_{d d}\left(\vec{r}_{i j_{D}}, \vec{\mu}_{i}, \vec{\mu}_{j}\right),
$$

where the Lennard-Jones potential is expressed in standard form:

$$
V_{L J}\left(r_{i_{\alpha}, j_{\beta}}\right)=4 \varepsilon\left[\left(\frac{\sigma}{r_{i_{\alpha}, j_{\beta}}}\right)^{12}-\left(\frac{\sigma}{r_{i_{\alpha}, j_{\beta}}}\right)^{6}\right],
$$

while the dipole-dipole potential is given by eq. (3), with $\mathbf{u}_{i j}=\vec{r}_{i j_{D}} / r_{i j_{D}}$. Here the vectors between molecular polarizability centers are expressed as

$$
\begin{aligned}
\vec{r}_{i_{\alpha}, j_{\beta}}= & \vec{r}_{i j}+l\left[\operatorname { c o s } \delta \left(\left\{\delta_{1, \beta}-\delta_{-1, \beta}\right\} \hat{\mathbf{c}}_{j}-\left\{\delta_{1, \alpha}\right.\right.\right. \\
& \left.\left.\left.-\delta_{-1, \alpha}\right\} \hat{\mathbf{c}}_{i}\right)+\sin \delta\left(\delta_{0, \alpha} \hat{\mathbf{b}}_{i}-\delta_{0, \beta} \hat{\mathbf{b}}_{j}\right)\right],
\end{aligned}
$$

where indices $\alpha, \beta=-1,0,1$ for lower, central and upper Lennard-Jones centers, respectively, and $l$ is the distance between two subsequent centers in a given molecule. The vector between the transverse dipoles of the molecules $i$ and $j$ is equal to

$$
\vec{r}_{i j_{D}} \equiv \vec{r}_{i_{0}, j_{0}}=\vec{r}_{i j}+l \sin \delta\left(\hat{\mathbf{b}}_{i}-\hat{\mathbf{b}}_{j}\right) .
$$

The interaction potential for any two molecules, eq. (12), can now be expanded in powers of $l / r_{i j}$ keeping the first nontrivial terms. In the mean-field approximation the pair interaction potential can be integrated over all intermolecular vectors $\vec{r}_{i j}$ within one smectic layer $[2,26]$ taking into account the steric cutoff at $\vec{r}_{i j}=\sigma$. To facilitate numerical calculations we also use the approximation of perfect orientational order of long molecular axes i.e. $\forall_{i, j}\left(\hat{\mathbf{c}}_{i} \cdot \hat{\mathbf{c}}_{j}\right) \cong 1$ and $\left(\hat{\mathbf{c}}_{i} \cdot \hat{\mathbf{b}}_{j}\right)=\left(\hat{\mathbf{b}}_{i} \cdot \hat{\mathbf{c}}_{j}\right) \cong 0$. As a result one obtains a simple pair potential, which is expressed as a sum of the polar and nonpolar contributions:

$$
\begin{aligned}
V(i, j)= & {\left[-3 J \sigma^{2} \lambda^{2}-\frac{\mu^{2}}{2 \sigma}\right]\left(\hat{\mathbf{b}}_{i} \cdot \hat{\mathbf{b}}_{j}\right) } \\
& +\frac{213}{2} \lambda^{4} J \sigma^{2}\left(\hat{\mathbf{b}}_{i} \cdot \hat{\mathbf{b}}_{j}\right)^{2},
\end{aligned}
$$

where $J$ is proportional to the $-\varepsilon, \mu$ is the value of electric dipole, $\sigma$ is the molecular diameter in the Lennard-Jones potential, and $\lambda=\frac{l}{\sigma} \tan \delta$. In the framework of the molecular field theory of smectic liquid crystals the one-particle distribution function is determined by the following selfconsistent equation (see for instance $[1,2,26,27]$ ):

$$
f_{1}(\hat{\mathbf{b}})=\frac{1}{Z} \exp \left[-\frac{U_{M F}(\hat{\mathbf{b}})}{k_{B} T}\right]
$$

where $Z$ is the one-particle partition function:

$$
Z=\int \exp \left[-\frac{U_{M F}(\hat{\mathbf{b}})}{k_{B} T}\right] \mathrm{d} \hat{\mathbf{b}}
$$

and where the mean-field potential $U_{M F}$ is determined self-consistently by the following expression:

$$
U_{M F}(\hat{\mathbf{b}})=\rho \int f_{1}\left(\hat{\mathbf{b}}_{2}\right) V\left(\hat{\mathbf{b}}, \hat{\mathbf{b}}_{2}\right) \mathrm{d} \hat{\mathbf{b}}_{2}
$$

where $\rho$ is the number density of molecules and $V\left(\hat{\mathbf{b}}, \hat{\mathbf{b}}_{2}\right)$ is the effective orientational pair interaction potential given by eq. (16). Substituting eq. (16) into eq. (19) one obtains the following explicit expression for the mean-field potential:

$$
\begin{aligned}
U_{M F}(i)= & \rho\left[-3 J \sigma^{2} \lambda^{2}-\frac{\mu^{2}}{2 \sigma}\right]\langle\cos (\phi)\rangle \cos (\phi) \\
& +\rho \frac{213}{4} \lambda^{4} J \sigma^{2}[1+\langle\cos (2 \phi)\rangle \cos (2 \phi)],
\end{aligned}
$$

where the angle $\phi$ is determined by the equation $\cos (\phi) \equiv$ $\hat{\mathbf{b}} \cdot \hat{\mathbf{m}}$, and where $\hat{\mathbf{m}}$ is the secondary director in the biaxial phases. One notes that the mean-field potential depends on the two orientational order parameters $\langle\cos (2 \phi)\rangle$ and $\langle\cos (\phi)\rangle$ which specify the nonpolar biaxial and polar order of short molecular axes, respectively. In the nonpolar biaxial phase only the order parameter $\langle\cos (2 \phi)\rangle$ is nonzero, while in the polar biaxial phase both order parameters do not vanish. After dropping the constant term in eq. (20), the dimensionless mean-field potential can be expressed as

$$
\begin{aligned}
V_{M F}(i) \equiv & \frac{U_{M F}(i)}{\rho \sigma^{2}|J|}=\left[3 \lambda^{2}-\frac{M}{2}\right]\langle\cos (\phi)\rangle \cos (\phi) \\
& -\frac{213}{4} \lambda^{4}\langle\cos (2 \phi)\rangle \cos (2 \phi)
\end{aligned}
$$




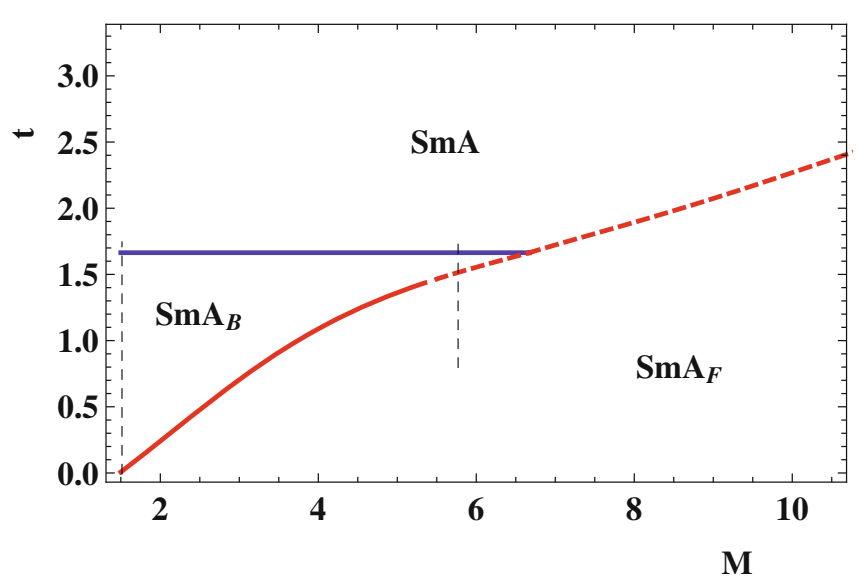

Fig. 2. (Color online) Phase diagram for $\lambda=\frac{1}{2}$. Dashed vertical lines represent the values of $M$ for which order parameters profiles are presented.

with $M=\frac{\mu^{2}}{\sigma^{3}|J|}$. Finally the mean-field free energy of the system is given by the following expression:

$$
\begin{aligned}
f_{\text {layer }} \equiv & \frac{F_{\text {layer }}}{\rho \sigma^{2}|J|}=\frac{1}{2}\left(\left[\frac{M}{2}-3 \lambda^{2}\right]\langle\cos (\phi)\rangle^{2}\right. \\
& \left.+\frac{213}{4} \lambda^{4}\langle\cos (2 \phi)\rangle^{2}\right)-t \ln (Z)
\end{aligned}
$$

where $t=\frac{k_{B} T}{\sigma^{2}|J|}$ is the reduced temperature in the system. One notes that in the limit of vanishing dipole moment this expression is reduced to the mean-field free energy of the nonpolar biaxial phases for system composed of bentcore molecules.

It should be noted that in this model we have considered only the dispersion and electrostatic dipolar interactions between central parts of bent-core molecules assuming that the polar part of this potential is responsible for the ordering of short molecular axes. Real bentcore molecules possess a much larger geometric anisotropy than that specified by the parameter $l / \sigma$ mainly due to the presence of long flexible chains. This large anisotropy stabilizes high orientational and translational order typical for smectic bent-core phases and determines the transition temperature into the smectic A phase.

\section{Results}

The values of order parameters as functions of temperature have been obtained by numerical minimization of the mean-field free energy, eq. (22), for various values of the coupling constants in eq. (21). Then the results have been summarized in the form of the reduced temperature $(t)$ and the reduced dipolar energy $(M)$ phase diagrams for two fixed values of $\lambda$. In order to provide credible results we have assumed that both contributions to the pair potential are comparable, and thus we present phase diagrams for $\lambda=\frac{1}{2}$ in fig. 2 , and $\lambda=\frac{1}{3}$ in fig. 3 . Both diagrams are qualitatively similar and contain the line of the

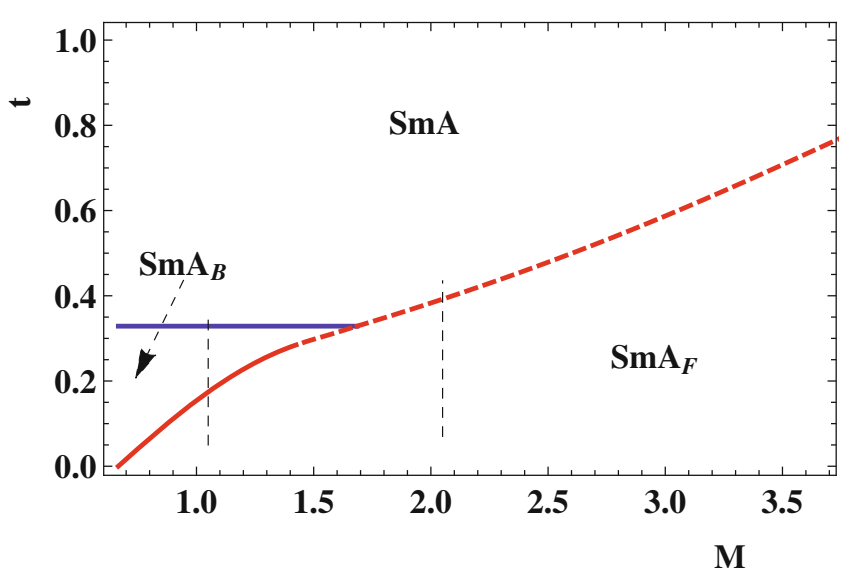

Fig. 3. (Color online) Phase diagram for $\lambda=\frac{1}{3}$. Dashed vertical lines represent the values of $M$ for which order parameters profiles are presented.
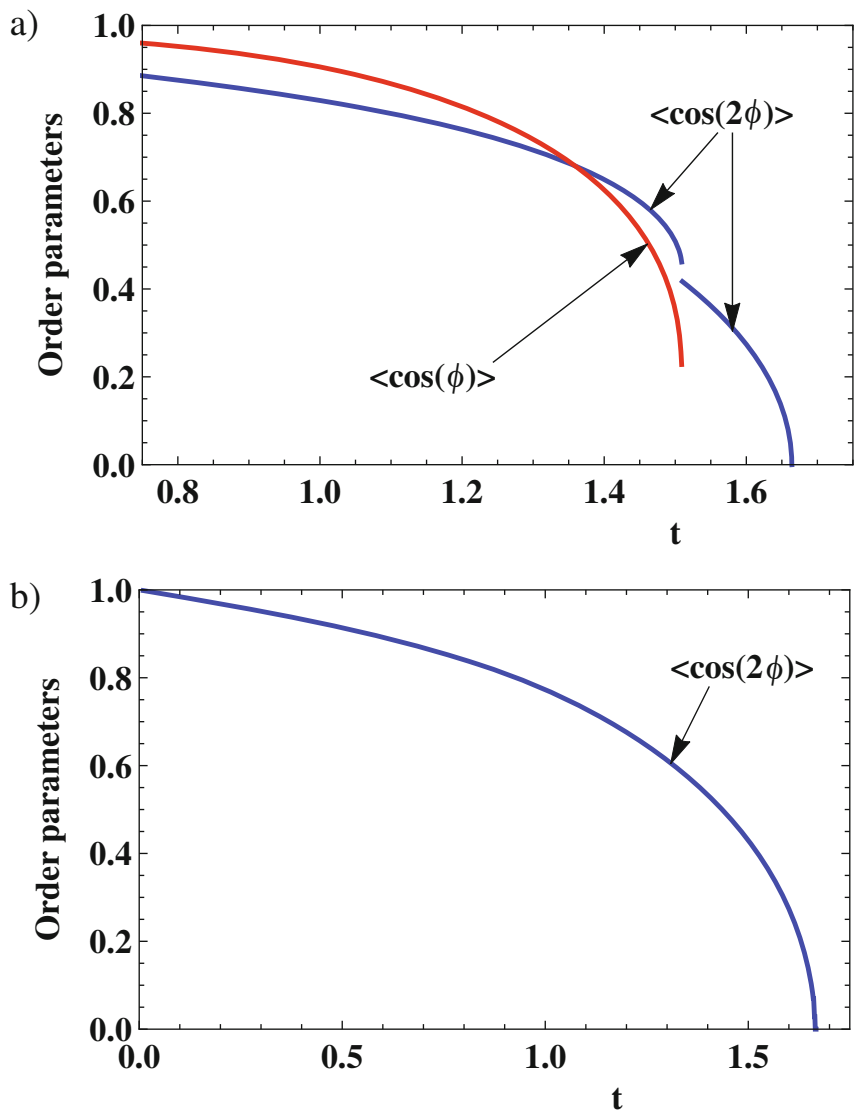

Fig. 4. (Color online) Temperature variation of the order parameters for $\lambda=\frac{1}{2}$ and for: $M=5.75$ (a), $M=1.5$ (b).

first-order transitions from the uniaxial smectic A phase to the ferroelectric phase (denoted as $\mathrm{SmA}_{F}$ ), and the line of second-order phase transition from the uniaxial smectic A phase to the biaxial nonpolar phase $\mathrm{SmA}_{B}$. Transition from the nonpolar $\mathrm{SmA}_{B}$ phase to the polar $\mathrm{SmA}_{F}$ phase can be either of the first or second order depending on the dipolar strength constant $M$. Representative order parameter profiles are shown in fig. 4 and fig. 5 . 
a)

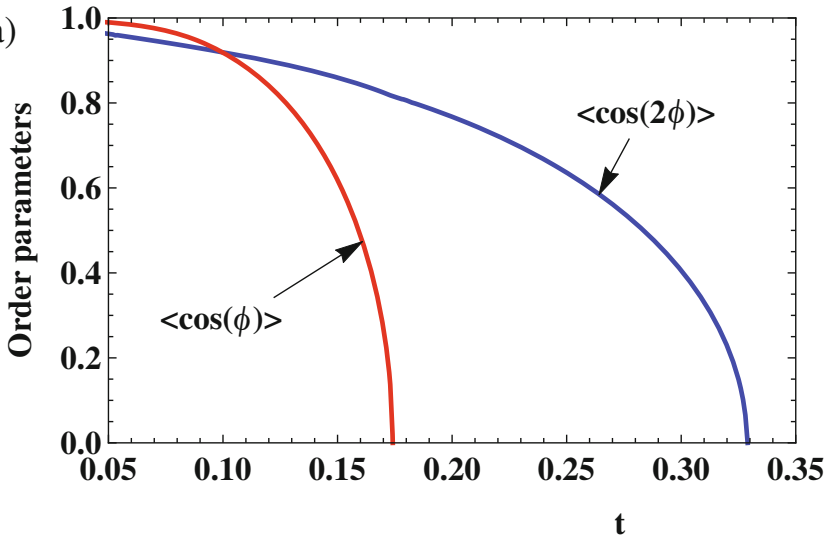

b)

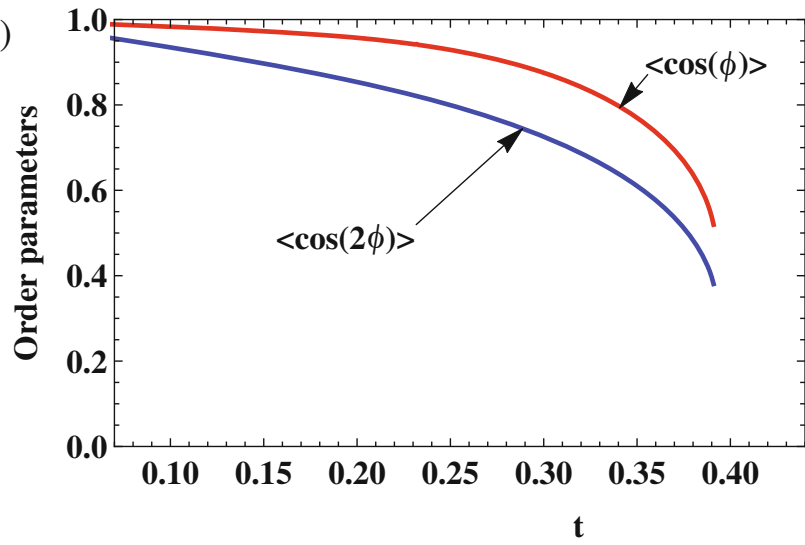

Fig. 5. (Color online) Temperature variation of the order parameters for $\lambda=\frac{1}{3}$ and for: $M=1.05$ (a), $M=2.05$ (b).

In both (a) panels of fig. 4 and fig. 5 the system undergoes the full sequence of phase transitions starting from the uniaxial smectic A phase thorough nonpolar biaxial smectic to the ferroelectric $\mathrm{SmA}_{F}$ phase.

Furthermore, one observes that for constant value of $\lambda$, when the reduced dipolar energy contribution described by $M$ is decreasing, the temperature range of polar $\mathrm{SmA}_{F}$ phase is shrinking, and finally is reduced to a point at a certain value of $M$. This limiting value of $M$ can formally be obtained from the condition that the average internal energy of the system:

$$
\frac{1}{2}\left(\left[\frac{M}{2}-3 \lambda^{2}\right]\langle\cos (\phi)\rangle^{2}+\frac{213}{4} \lambda^{4}\langle\cos (2 \phi)\rangle^{2}\right)
$$

is positively defined. This constraint in formula (23) implies that $\left[\frac{M}{2}-3 \lambda^{2}\right] \geq 0$, which for fixed $\lambda$ yields the following lover limit for the dipolar coupling constant: $M \geq 6 \lambda^{2}$. In the case of equality only the nonpolar $\mathrm{SmA}_{B}$ phase is stable at nonzero temperatures, and the temperature variation of the order parameter is shown in fig. 4(b). The order parameter profiles for the opposite situation are presented in fig. 5(b). In this case the system undergoes the direct uniaxial smectic A to the polar $\mathrm{SmA}_{F}$ phase transition, and there is no nonpolar $\mathrm{SmA}_{B}$ phase. Further increase of the dipole strength $M$ results in a weakening of the first-order phase transition.
One can readily see that the transition into the ferroelectric phase occurs at reduced temperatures $t \sim 1$ when the dimensionless parameter $M$ is in the range from 2 to 10. This means that Lennard-Jones coupling constant $J$ must be of the order of $k_{B} T$, and the dipole-dipole interaction potential (proportional to $\mu^{2} / \sigma^{3}$ ) at a contact distance should be of the order of $(2-10) k_{B} T$ which is consistent with the realistic values of the molecular dipole at the contact distance $\sigma$.

\section{Discussion}

It has been shown in this paper that the electrostatic dipole-dipole interaction may promote ferroelectric ordering in systems with two-dimensional restricted geometry like smectic liquid crystals with high positional order. In $3 \mathrm{D}$ polar fluids without any translational order the long nature of the dipole-dipole interaction results in a dependence of the dipole-dipole contribution to the free energy on the sample shape and on the boundary conditions. In contrast, in quasi-two-dimensional systems the contribution from the dipole-dipole interaction to the total free energy is regular and does not depend either on the shape of the sample or on the dielectric constant of the surrounding medium. This enables one to explain why ferroelectric ordering is more likely to be observed in layered systems like bent-core smectic liquid crystals and not in conventional polar fluids or nematic liquid crystals composed of polar molecules. This conclusion has been justified in sect. 2 assuming that the centers of molecules are confined to a plane. However, one expects that similar arguments would apply if the molecular centers are confined to a finite layer. Thus the present theory may also be generalized taking into account some translational fluctuations.

One notes that the ferroelectric ordering in orthogonal bent-core smectic phases, which has recently been observed experimentally, is further stabilized by the high orientational order of long molecular axes (see a recent paper [14] where high values of the nematic order parameter have been measured). In the case of high nematic order of long molecular axes the transverse dipoles of bent-core molecules are restricted to the plane of the smectic layer. This reduces the orientational entropy which opposes the polar order.

In the case of bent-core molecules polar intermolecular interactions are determined both by the permanent molecular dipoles and by the intermolecular attraction modulated by polar molecular shape. It has been shown that the latter interaction promotes the antiparallel orientation of the steric dipoles of the neighboring bent-core molecules which stabilizes the nonpolar biaxial Smectic A phase. As a result the ferroelectric ordering may appear due to a subtle balance between the dipole-dipole and steric intermolecular interactions, and the system generally may exhibit both phases at different temperatures.

Two phase diagrams have been presented which illustrate the dependence of the transition temperatures between different phases on the reduced molecular dipole for different values of the molecular bent angle. In general 
the increase of the molecular dipole leads to a stabilization of the ferroelectric phase while the region of stability of the nonpolar biaxial phase is increasing with the increasing bent angle, at least when the angle is not too large. Transition from the uniaxial smectic A phase to the ferroelectric $\mathrm{SmA}_{F}$ is of the first order while the transition from the uniaxial smectic A phase to the biaxial nonpolar $\mathrm{SmA}_{B}$ is of the second order. Transition from the nonpolar $\mathrm{SmA}_{B}$ phase to the polar $\mathrm{SmA}_{F}$ phase can be either of the first or of the second order depending on the dipolar strength constant $M$. Representative order parameter profiles in both phases have also been calculated numerically.

One notes that the phase diagrams presented in this paper are qualitatively similar to the diagrams obtained using the theory of orientational phase transitions in molecular crystals with polar and quadrupolar interactions [28]. This similarity is related to the mean-field character of both theories (including the two-particle cluster approximation also used in [28]) which results in a similar mathematical form of the free energy. At the same time the molecular origin of the ferroelectric ordering in both cases is significantly different. The theory of phase transitions in molecular crystals [28] is based on a simple model short-range polar interaction potential which is not directly related to the dipole-dipole interaction. In contrast, the present theory employs the long-range dipole-dipole interaction potential which makes a regular contribution to the free energy (which is independent of the boundary conditions) only in strongly layered phases, i.e. in quasitwo-dimensional systems.

It should be noted that the theory presented in this paper is based on the assumption that the director is perpendicular to the smectic plane. Thus only orthogonal smectic phases have been taken into consideration. It is well known, however, that many bent-core liquid crystals exhibit also a sequence of tilted ferro- and ferri-electric phases. These tilted phase can be described using the same general method which has been used in the molecular theory of de Vries like smectics C $[26,29,30]$. The corresponding theory will be presented in our future publication.

This work is supported by the EPSRC grant (EP/H046941/1) which is a part of the Materials World Network Project. One of us (G.P.) also acknowledges a partial support from European Union through the European Social Fund within "Cracow University of Technology development program - top quality teaching for the prospective Polish engineers; University of the 21st century" project (Contract No. UDA-POKL.04.01.01-00029/10-00).

Open Access This is an open access article distributed under the terms of the Creative Commons Attribution License (http://creativecommons.org/licenses/by/4.0), which permits unrestricted use, distribution, and reproduction in any medium, provided the original work is properly cited.

\section{References}

1. J.W. Goodby S.A. Pikin, R. Blinc, N.A. Clark, S.T. Lagerwall, M.A. Osipov, T. Sakurai, K. Yoshino, B. Zeks, Ferroelectric Liquid Crystals: Principles, Properties and Applications in Ferroelectricity and Related Phenomena Series, Vol. 7 (Gordon \& Breach Science Publishers, 1992).

2. M.A. Osipov, M.V. Gorkunov, Phys. Rev. E 77, 031701 (2008).

3. B. Groh, S. Dietrich, Phys. Rev. E 50, 3814 (1994)

4. M.A. Osipov, P.I.C. Teixeira, M.M. Telo da Gama, J. Phys. A 30, 1953 (1997).

5. F. Bisi, A.M. Sonnet, E.G. Virga, Phys. Rev. E 82, 041709 (2010).

6. S. Giura, B.G. Markus, S.H.L. Klapp, M. Schoen, Phys. Rev. E 87, 012313 (2013).

7. T. Watanabe, S. Miyata, T. Furukawa, H. Takezoe, T. Nishi, M. Sone, A. Migita, J. Watanabe, Jpn. J. Appl. Phys. 35, L505 (1996).

8. A. Eremin, S. Diele, G. Pelzl, H. Nadasi, W. Weissflog, J. Salfetnikova, H. Kresse, Phys. Rev. E 64, 051707 (2001).

9. B.K. Sadashiva, R.A. Reddy, R. Pratibha, N.V. Madhusudana, J. Mater. Chem. 12, 943 (2002).

10. S.T. Wang, X.F. Han, A. Cady, Z.Q. Liu, A. Kamenev, L. Glazman, B.K. Sadashiva, R.A. Reddy, C.C. Huang, Phys. Rev. E. 70, 061705 (2004).

11. B. Glettner, S. Hein, R.A. Reddy, U. Baumeister, C. Tschierske, Chem. Commun. 25, 2596 (2007).

12. C. Keith, M. Prehm, Y.P. Panarin, J.K. Vij, C. Tschierske, Chem. Commun. 46, 3702 (2010).

13. R.A. Reddy, Ch. Zhu, R. Shao, E. Korblova, T. Gong, Yo. Shen, E. Garcia, M.A. Glaser, J.E. Maclennan, D.M. Walba, N.A. Clark, Science 332, 72 (2011).

14. S. Sreenilayam, Y.P. Panarin, J.K. Vij, M. Osipov, A. Lehmann, C. Tschierske, Phys. Rev. E 88, 012504 (2013).

15. Y.P. Panarin, M. Nagaraj, S. Sreenilayam, J.K. Vij, A. Lehmann, C. Tschierske, Phys. Rev. Lett. 107, 247801 (2011).

16. D. Pociecha, M. Čepič, E. Gorecka, J. Mieczkowski, Phys. Rev. Lett. 91, 185501 (2003).

17. D. Pociecha, E. Gorecka, M. Čepič, N. Vaupotič, K. Gomola, J. Mieczkowski, Phys. Rev. E 72, 060701 (2005).

18. M.A. Osipov, Molecular theories of liquid crystals in Handbook of Liquid Crystals, edited by John W. Goodby, Peter J. Collings, Takashi Kato, Carsten Tschierske, Helen Gleeson, Peter Raynes, Vol. 1 (Wiley VCH, Weinheim, 2014).

19. W.M. Gelbart, B. Barboy, Acc. Chem. Res. 13, 290 (1980).

20. J. Peláez, M.R. Wilson, Phys. Rev. Lett. 97, 267801 (2006).

21. F. Bisi, R. Rosso, E.G. Virga, G.E. Durand, Phys. Rev. E 78, 011705 (2008).

22. F. Bisi, R. Rosso, Eur. J. Appl. Math. 23, 29 (2012).

23. M.A. Osipov, P.I.C. Teixeira, M.M. Telo da Gama, Phys. Rev. E 54, 2597 (1996).

24. J.D. Jackson, Classical Electrodynamics, 2nd edition (Wiley, New York, 1975), p. 139.

25. M.A. Osipov, G. Pająk, J. Phys.: Condens. Matter 24, 142201 (2012).

26. M. Osipov, G. Pająk, Phys. Rev. E. 85, 021701 (2012).

27. G. Pająk, M.A. Osipov, Phys. Rev. E. 88, 012507 (2013).

28. T.J. Krieger, H.M. James, J. Chem. Phys. 22, 796 (1954).

29. M.V. Gorkunov, M.A. Osipov, J.P.F. Lagerwall, F. Giesselmann, Phys. Rev. E 76, 051706 (2007).

30. M.V. Gorkunov, M.A. Osipov, J. Phys.: Condens. Matter 20, 465101 (2008). 